\title{
Cleaning Matters!
}

Ahmed Salaheldin Mahmoud ${ }^{1}$ and Doris Segets ${ }^{2 *}$

1: Institute of Particle Technology (LFG), Interdisciplinary Center for Functional Particle Systems (FPS), Friedrich-Alexander-Universität Erlangen-Nürnberg (FAU), Cauerstraße 4, 91058 Erlangen, Germany

2: Process Technology for Electrochemical Functional Materials, Institute for Combustion and Gas Dynamics-Reactive Fluids, and Center for Nanointegration Duisburg-Essen (CENIDE), University of Duisburg-Essen (UDE), Carl-Benz-Straße 199, 47057, Duisburg, Germany

*: Correspondence to doris.segets@uni-due.de

\section{Outline}

SI 1 Automated setup _ (p. 2)

SI 2 Absorbance spectroscopy

SI 3 Automated synthesis

SI 4 Comparison of cleanliness

SI 5 Semiautomated protocol with inefficient manual post-processing (p. 5) 


\section{SI 1 Automated setup}

The automated platform was a Chemspeed(C) Technologies (CST) Swing XL Autoplant II Hybrid. A detailed description of the platform equipment can be found in our previous work. ${ }^{11}$ Briefly, the robotic platform is enclosed inside an MBraun Glovebox equipped with a purifier that keeps the oxygen and humidity values to a minimum ( $\leq 20 \mathrm{ppm}$ per box volume). The platform is further equipped with a liquid handling tool known as the four needle head (4NH) that performs liquid transfers from any position in the platform to another and that can be lowered with an accuracy of $0.2 \mathrm{~mm}$. The $4 \mathrm{NH}$ is connected to a diluter pump that consists of four syringes, two $1 \mathrm{~mL}$ and two $10 \mathrm{~mL}$ syringes with an error of $\pm 1.5 \%$ of the maximum volume. The $4 \mathrm{NH}$ performs a rinsing step between volumetric transfer tasks either internally, by running a predefined volume of solvent via the dilutor pumps or externally, by insertion of the needles into a pocket where empty solvent flows internally and externally. In the middle of the platform three rack holders, one for holding glass vials of volumes up to $60 \mathrm{~mL}$, a plate holder for microtiter plates (MTPs) or deep well plates (DWPs) and a heatable shaking rack for holding glass vials and for the preparation of small stock solutions and vial storage are installed. The heart of the robot is the reactor module that consists of six reactors with a total volume of $100 \mathrm{~mL}$ and six feed vessels with a total volume of $50 \mathrm{~mL}$ arranged in parallel. The reactors provide electrical heating with automatic temperature control up to $340{ }^{\circ} \mathrm{C}$ at $0.1{ }^{\circ} \mathrm{C}$ resolution assured by in situ Pt100 temperature probes. Stirring up to $1600 \mathrm{rpm}$ can be realized with anchor or triple blade stirrers, depending on the chosen flow field, while the feed vessels are stirred with magnetic stirrer bars (up to $1400 \mathrm{rpm}$ ). 


\section{SI 2 Absorbance spectroscopy}

Absorbance spectroscopy on CdSe quantum dots was performed using a BioTek@ Synergy MX microtiter plate (MTP) reader using 96 well MTPs made from polystyrene (Rotalibo®). For details we refer to our previous work. ${ }^{1}$ Spectral scanning was performed from $700 \mathrm{~nm}$ to $300 \mathrm{~nm}$ at $1 \mathrm{~nm}$ steps.

\section{SI 3 Automated synthesis}

For robotic synthesis, Cd and Se stock solutions were first prepared in one reactor and one feed vessel. The preparation steps were as follows:

i) For the manual protocol, $195 \mathrm{mg}$ of Cadmium oxide (CdO) was weighed and transferred to a reactor and $225 \mathrm{mg}$ of Se was weighed and transferred to a feed vessel. To avoid effects of ionization due to the low glovebox humidity, a deionizer was positioned inside of the glovebox, close to the balance, the reactor and the feed vessel. After successfully transferring the powders, $6 \mathrm{~mL}$ of oleic acid (OLA) were transferred to the reactor via a manual syringe and $3 \mathrm{~mL}$ of trioctylphosphine (TOP) to the feed vessel. Then, $37.5 \mathrm{~mL}$ of ODE were automatically transferred to the reactor and another $37.5 \mathrm{~mL}$ to the feed vessel subsequently. An external rinsing step was performed between the two latter tasks to avoid cross-contamination.

ii and iii) For the semiautomated and fully automated protocols, $392.5 \mathrm{mg}$ of CdO was weighed and transferred to a reactor and $225 \mathrm{mg}$ of Se was weighed and transferred to a feed vessel. To avoid effects of ionization due to the low glovebox humidity, a deionizer was positioned inside of the glovebox, close to the balance, the reactor and the feed vessel. After successfully 
transferring the powders, $18 \mathrm{~mL}$ of OLA were transferred to the reactor via a manual syringe and $3 \mathrm{~mL}$ of TOP were transferred to the feed vessel via a manual syringe. Finally, $56.25 \mathrm{~mL}$ of ODE were added automatically to the reactor and $9.375 \mathrm{~mL}$ ODE were added to the feed vessel, again with an external rinsing step between the two tasks to avoid cross contamination.

After these preparations, in all cases i-iii), the reactor was kept open and heated to $160{ }^{\circ} \mathrm{C}$ and stirred with an anchor stirrer at $150 \mathrm{rpm}$ to convert CdO to Cd-OLA. Meanwhile, the feed vessel was stirred at $600 \mathrm{rpm}$ at room temperature to convert Se to TOP-Se. Both, the reactor and the feed vessel were stirred overnight $(\sim 8 \mathrm{~h})$.

After the preparation of the stock solutions, usually four other reactors were filled with $15 \mathrm{~mL}$ of ODE and heated to $160{ }^{\circ} \mathrm{C}$. Then, $2.9 \mathrm{~mL}$ (5.8 mL for manual protocol i)) of Cd-OLA were transferred from the stock solution reactor to every synthesis reactor with an external rinsing step between each transfer. After that, each reaction was executed in series where the temperature set point was raised to $260{ }^{\circ} \mathrm{C}$ and kept there for $10 \mathrm{~min}$ for temperature equilibration. The $4 \mathrm{NH}$ was then programmed to withdraw $0.5 \mathrm{~mL}$ ( $2 \mathrm{~mL}$ for protocol i)) of TOP-Se from the feed vessel with the stock solution, prior moving inside the reactor and quickly injecting the TOP-Se educt into the reaction mixture. This initialized the CdSe reaction and subsequent QD formation.

Then, all four needles of the 4NH were used to quickly withdraw samples during early stages of the reaction. The times of sample withdrawal were $6 \mathrm{~s}, 27 \mathrm{~s}, 49 \mathrm{~s}$ and $70 \mathrm{~s}$. Afterwards, the first four CdSe samples were transferred to predefined wells in a MTP. Two more samples were withdrawn at $430 \mathrm{~s}(\sim 7 \mathrm{~min})$ and $650 \mathrm{~s}(\sim 11 \mathrm{~min})$. After withdrawal of the final sample, the next reactor repeated the same steps. 
After completion of all, typically four, reactions, the run was finished and the reactors were left to cool down prior the respective cleaning procedures were initiated. Finally, the MTP with the samples was transferred to the plate reader for HT characterization.

\section{SI 4 Reactor components and cleanliness}

Figure S1a-c visualizes in detail the reactor components that required cleaning, including triple blade stirrer and reactor bottom (a), magnetic holder (b) and the reactor top with the temperature probe (c). Moreover, Figure S1d-e visualizes a still dirty reactor with colored residuals (d) and a cleaned reactor (e).

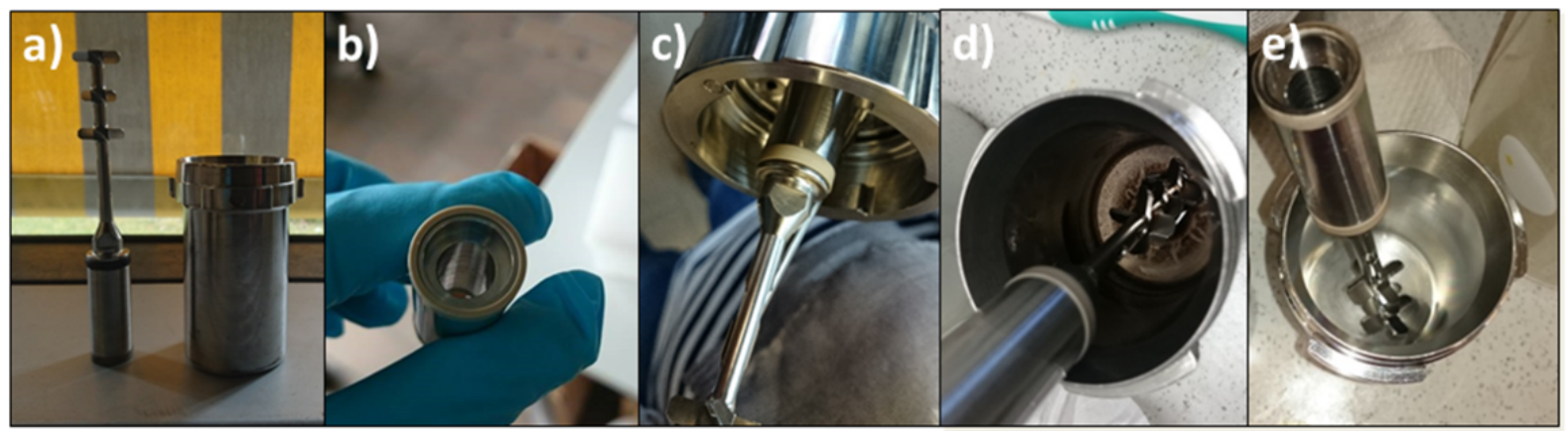

Figure S1. Reactor components that require cleaning including a) a triple blade stirrer (left) and the reaction vessel (right), b) magnetic stirrer coupling, c) view into a reactor and temperature probe, images of $d$ ) an uncleaned reactor and e) a cleaned reactor. 


\section{SI 5 Semiautomated protocol with inefficient post-processing}

Figure S2 shows the effect of residuals like toluene, isopropanol or soap caused by inadequate manual post-processing after semiautomated cleaning on the reproducibility of CdSe nanoparticle synthesis. This highlights that special care on all details during the manual part of the cleaning procedure is required if a semiautomated cleaning is performed.

a)

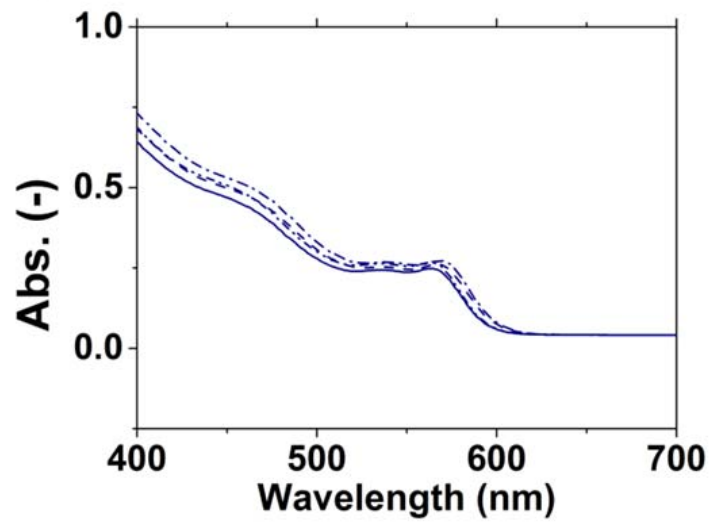

b)

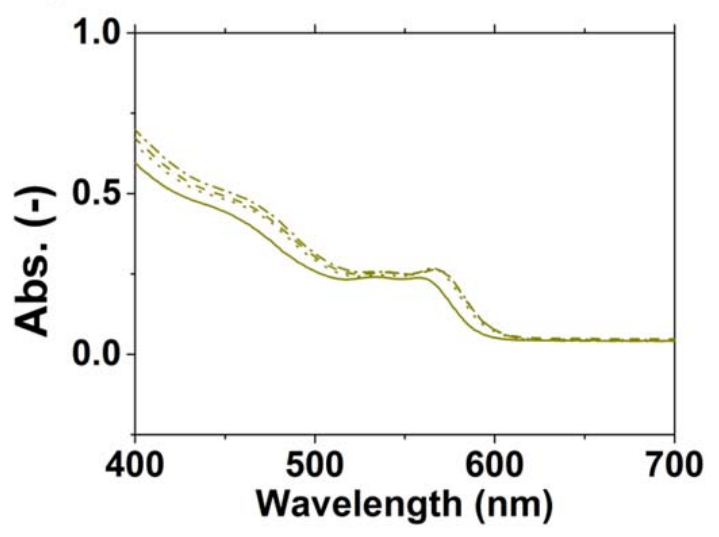

Figure S2. Effect of residuals from inefficient cleaning on the resulting CdSe quantum dots withdrawn after $70 \mathrm{~s}$ for two different automated runs (4 samples for each run).

\section{Literature}

1. Salaheldin, A. M.; Walter, J.; Herre, P.; Levchuk, I.; Jabbari, Y.; Kolle, J. M.; Brabec, C. J.; Peukert, W.; Segets, D., Automated synthesis of quantum dot nanocrystals by hot injection: Mixing induced self-focusing. Chemical Engineering Journal 2017, 320, 232-243. 